\title{
A New Design for All-Normal Near Zero Dispersion Photonic Crystal Fiber with Selective Liquid Infiltration for Broadband Supercontinuum Generation at $1.55 \mu \mathrm{m}$
}

\author{
Partha Sona Maji and Partha Roy Chaudhuri \\ Department of Physics \& Meteorology, Indian Institute of Technology, Kharagpur 721 302, India \\ Correspondence should be addressed to Partha Sona Maji; parthamaji.1984@gmail.com
}

Received 21 December 2013; Revised 20 March 2014; Accepted 21 March 2014; Published 16 April 2014

Academic Editor: Luciano Mescia

Copyright ( 2014 P. S. Maji and P. Roy Chaudhuri. This is an open access article distributed under the Creative Commons Attribution License, which permits unrestricted use, distribution, and reproduction in any medium, provided the original work is properly cited.

\begin{abstract}
A new design of all-normal and near zero flattened dispersion based on all-silica photonic crystal fibers (PCFs) using selectively liquid infiltration technique has been proposed to realize smooth broadband supercontinuum generation (SCG). The investigation gives the details of the effect of different geometrical parameters along with the infiltrating liquids on the dispersion characteristics of the fiber. Numerical investigations establish a dispersion value of $-0.48 \mathrm{ps} / \mathrm{nm} / \mathrm{km}$ around the wavelength of $1.55 \mu \mathrm{m}$. The optimized design has been found to be suitable for SCG around the C band of wavelength with flat broadband wavelength band ( $375 \mathrm{~nm}$ bandwidth) and smooth spectrum with only a meter long of the PCF. The proposed structure also demonstrates good tunable properties that can help correct possible fabrication mismatch towards a better optimization design for various optical communication systems.
\end{abstract}

\section{Introduction}

Broadband smooth flattened supercontinuum generation (SCG) has been the target for the researchers for its enormous applications in the field of metrology, optical sensing, optical coherence tomography, wavelength conversion, and so forth [1]. Recent developments for mid-infrared (MIR) SC source have been investigated based on nonsilica fibers [2, 3]. Unconventional PCFs based on aperiodic structure have also been investigated [4]. However, achieving flat broadband smooth SC sources for IR applications remains challenging [1]. One of the foremost requirements of generating broadband flattened SCG is to achieve near zero flattened dispersion around a targeted wavelength. Photonic crystal fibers (PCFs) $[5,6]$, which enjoys some unique properties like wide band single mode operation, great controllability over dispersion properties, and higher nonlinearity, has been the target host for SCG for the last decades [1]. Researchers have worked on designing novel dispersion profiles with variable air-hole diameter in the cladding [7-13] and this design can be further manipulated for SC generation by pumping at the near zero wavelength $[9,14]$. However, the realizing technology of complicated structures or PCF having air-holes of different diameters in microstructure cladding remains truly challenging. An alternative route of achieving similar performance is shown to be practicable by filling the air-holes with liquid crystals $[15,16]$ or by various liquids such as polymers [17], water [18], and ethanol [19]. Tunable photonic band gap (PBG) effect and long-period fiber grating have been successfully realized with liquid-filled PCFs [20]. Theoretical design of dispersion management by filling the air-holes of the PCF with selective liquids for both single wavelength and broadband wavelength applications has been studied [21, 22]. In the present work, a new design of silica based PCF with all-normal near zero chromatic dispersion around the desired wavelength of $1.55 \mu \mathrm{m}$ through selectively liquid-filled inner air-holes has been proposed and the optimized design has been targeted for achieving smooth and flattened broadband SC spectra. As most of the power resides within the core of the PCF, liquid infiltration in air-holes will have the only effect in dispersion controllability. We also investigate the temperature dependence of the liquid-filled PCFs for better optimization 
design with its tunable property of shifting dispersion peak for optical communication systems. The main advantage with this approach is that we need not require different air-holes in the cladding to realize near zero dispersion at the desired wavelength; also we can tune the dispersion towards a better optimized design by changing the temperature of the liquid and thereby achieving highly smooth and flat broadband SC spectra with only a meter long of the fiber.

\section{Liquid-Filled Photonic Crystal Fiber and Related Issues}

Conventional PCFs have cladding structures formed by airholes with the same diameter arranged in a regular triangular or square lattice. By altering the PCF geometrical parameters, namely, hole-to-hole spacing $(\Lambda)$ and air-hole diameter $(d)$, the modal properties, in particular, the dispersion properties can be easily engineered $[5,6]$. However, the dispersion slope of such PCFs having air-holes of the same diameter cannot be tailored in a wide wavelength range. The current research aims to achieve all-normal dispersion close to zero at a desired wavelength with a flat slope of the dispersion curve using regular triangular-lattice structure having airholes of the same size uniformly distributed in the cladding. A common route to realize these goals of flat dispersion around a specific wavelength is by varying the size of airholes in different layers and is well known in [7-12]. Because of the fabrication limitation, the concept finds limited use as a practical fiber. This research looks for the achievement of these targets through a regular conventional PCF by incorporating the effects of filling air-hole ring with a liquid of predetermined refractive index (RI). Depending upon the refractive indices of the infiltrating liquid to the air-holes, the local refractive indices of the layer can be modified. In that way, the effect of variable air-hole diameter can be realized by infiltrating the air-holes with liquids of different refractive indices. Depending upon the RI of the infiltrating liquid, we could have the effect of different air-hole diameter.

The fabrication of such a fiber is simplified due to the uniformity of the air-holes in the cladding. To manufacture these PCFs, one must first selectively block specified air-holes and infuse the liquid into the unblocked holes. One possible way is to employ the fusion splicing technique [18-20]. The inner ring of the air-holes can be infiltrated with liquid, first by fusing the outer rings of air-holes with tailored electric arc energies and fusion times [23] and then by immersing one end of the fiber in a liquid reservoir and applying vacuum to the other end of the fiber [18]. This can be a possible way to infiltrate liquid in our case. Another way of selective plug specified air-hole layers in the PCFs is to use microscopically positioning tips with glue [24]. Not only airhole layers but also a single air-hole can be easily blocked by using this technique. In spite of the above methods, one can also selectively infiltrate the liquid into specified airhole layers from a macroscopic fiber preform to a connected microstructured PCF by using an applied pressure [21]. With the optimized air-hole diameters of the order of $0.60 \mu \mathrm{m}$, it should be easy to collapse the air-holes and fill the liquid into the air-holes with such dimensions. It is also interesting to note that PCFs with similar or smaller air-hole diameter was realized [25], making the proposed design practically achievable. With the advancement of technology, a good precision of the PCF parameters can be easily implemented.

There are certain issues related to the infiltration of liquid to the air-holes, such as whether the fluid wets glass and how viscous it is. If the liquid does not wet glass, then surface tension will oppose entry of the liquid into the air-hole, thereby making the process difficult to realize. The required pressure to push such a liquid into the air-holes can be worked out with the value of its surface tension and contact angle. With an air-hole diameter of the order of $0.60 \mu \mathrm{m}$, pressure by a vacuum pump will be sufficient.

If the fluid does wet glass, then the air-hole should be filled but the filling-up speed will depend upon viscosity. It can be worked out how quickly the air-holes will be filled using the expressions for Poiseuille flow in a pipe [26]. In other words, the air-holes can be filled (and how quickly), with the given values for surface tension, contact angle, and viscosity. With the fast advancement of the technology, submicron filling of air-holes will not be very difficult to achieve.

The selective hole filling technique provides a couple of advantages. Firstly, all the air-holes are of the same diameter, which is easier to be fabricated compared to fibers with multiple different submicron air-hole sizes. Secondly, a regular PCF with selective infiltration with a liquid provides huge flexibility for tremendous applications. This is why the paper pursues selective air-hole filling approach towards the target of all-normal near zero dispersion in a microstructured optical fiber. Notably, the technique comes out in designing fibers for various other applications [21, 22, 27-32].

\section{Analysis Method and Design Optimization of the PCF}

As an initial attempt, we consider a regular PCF with four rings of air-holes with $\mathrm{C}_{6 \mathrm{v}}$ symmetry with the central air-hole missing like normal index-guiding PCF as shown in Figure 1. The inner ring of air-holes is infiltrated with a liquid of certain RI's. Throughout our design study we have kept higher air-filing fraction, thereby reducing the confinement loss of the structure. As can be seen from our numerical results, we need only a meter long of such a fiber; the propagation loss of the high power spectrum can therefore be highly reduced. By optimizing the RI value $\left(n_{L}\right)$ of the infiltrating liquid combined with PCF geometrical parameters, namely, pitch $(\Lambda)$ and air-hole diameter $(d)$, all-normal near zero dispersion around a particular wavelength can be realized.

3.1. Dispersion Analysis. Modal fields as well as dispersion parameters are calculated by using commercially available CUDOS software along with MATLAB for numerically calculating the dispersion relation. The total dispersion $(D)$ is computed with [33]:

$$
D=-\frac{\lambda}{c} \frac{d^{2} \operatorname{Re}\left[n_{\mathrm{eff}}\right]}{d \lambda^{2}} .
$$




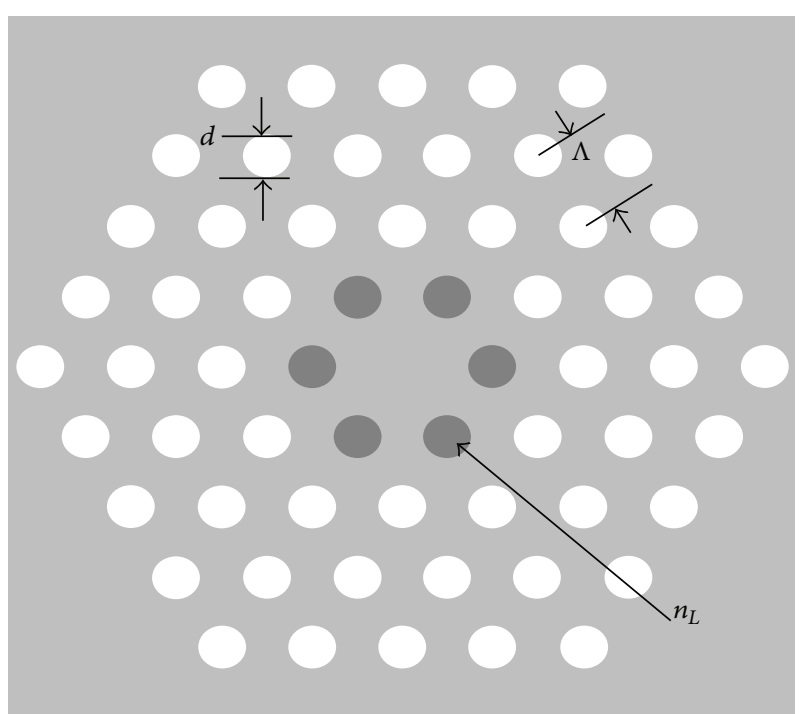

Figure 1: Cross section of the proposed photonic crystal fiber. The shaded regions represent air-holes infiltrated with liquid with refractive indices $n_{L}$.

Here $\operatorname{Re}\left[n_{\text {eff }}\right]$ stands for the real part of the effective indices, $c$ is the speed of light in vacuum, and $\lambda$ is the wavelength.

3.2. Supercontinuum Generation (SCG) Analysis. The SCG with the liquid filled near zero all-normal dispersion silica based PCF for smooth flattened broadband spectra has been studied by solving the nonlinear Schrödinger equation (NLSE) [33] describing the pulse propagation through the fiber length. The NLSE takes into account the contributions of the linear effects (attenuation, chromatic dispersion, and high-order dispersions) and the nonlinear effects (selfphase modulation, stimulated Raman scattering, and selfsteepening). NLSE for slowly varying pulse envelope $A(z, T)$ in the retarded time frame $T$ is given through (2) as follows:

$$
\begin{aligned}
\frac{\partial A}{\partial Z}+ & \frac{\alpha}{2} A-\sum_{n \geq 2} \frac{i^{n+1}}{n !} \beta n \frac{\partial^{n} A}{\partial T^{n}} \\
= & i \gamma\left(1-f_{R}\right)\left([A]^{2} A-\frac{i}{w_{0}} \frac{\partial}{\partial T}\left([A]^{2} A\right)\right) \\
& +i \gamma f_{R}\left(1+\frac{i}{w_{0}}\right)\left(A \int_{0}^{\infty} h_{g}(\tau)|A(z, T-\tau)|^{2} \partial \tau\right),
\end{aligned}
$$

where $\alpha$ is the loss coefficient, $\beta_{n}$ are the $n$th order dispersion, $\omega_{0}$ is the input pulse frequency, $\tau$ is the present time frame, and $f_{R}$ is the fractional contribution due to delayed Raman function $h_{g}(\tau)[33,34]$. The nonlinear coefficient $\gamma$ is given by (3) as follows:

$$
\gamma=\frac{2 \pi n_{2}}{\lambda A_{\text {eff }}}
$$

where $n_{2}$ is the nonlinear refractive indices of silica and $A_{\text {eff }}$ is the effective area at the pumping wavelength $\lambda$.

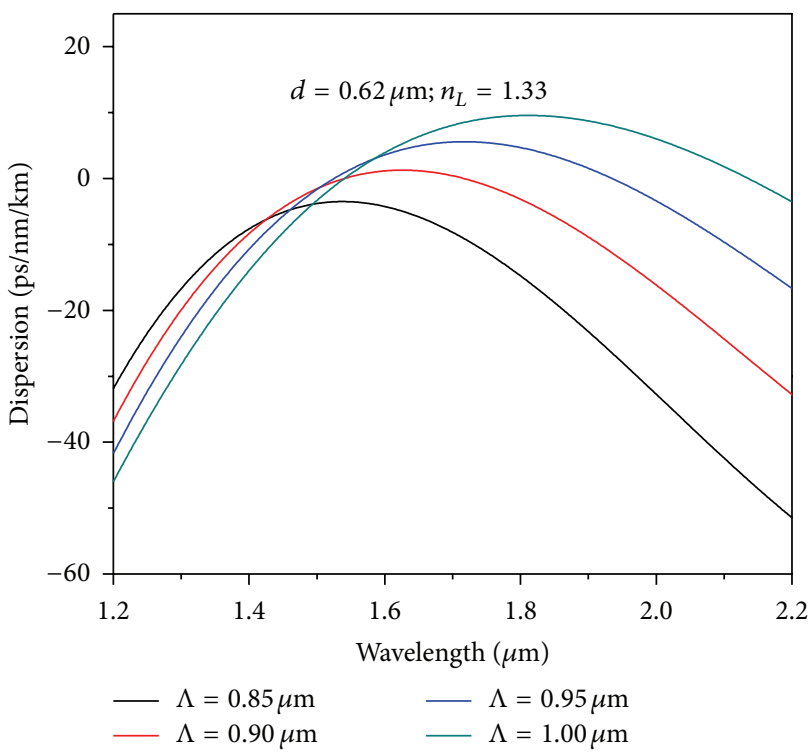

FIgure 2: Computed dispersion of the PCF as a function of pitch $(\Lambda)$ keeping $n_{L}$ and $d$ fixed.

3.3. Optimization for All-Normal Near Zero Dispersion at the Target Wavelength. The nonlinear effects in a PCF strongly depend on the dispersive characteristic and the length of the employed optical fiber [1]. In particular, with reference to SCG, the shape of the total dispersion curve can be tailored to obtain a broadband output spectrum. The approach of the present research of optimization towards designing near zero all-normal flat dispersion relies on varying multidimensional parameter space that consists of the liquid RI $\left(n_{L}\right)$, the pitch $\Lambda$, and air-hole diameter $(d)$. The procedure is followed in three steps. In the first step, the sensitivity of the variation of the parameters towards the total dispersion has been illustrated by varying one of the design parameters on the dispersion keeping other parameters constant. In the second stage, we took one of the parameters fixed (value obtained from the first step) and optimized the other parameters towards the target. Once one parameter is optimized, we reoptimize the design by adjusting other parameters. In the third stage we select a practical liquid (wavelength dependent RI) close to the optimized RI and optimize the other parameters to achieve the target of all-normal near zero dispersion. The first stage of the design optimization is presented as follows. Figure 2 shows the effect of $\Lambda$ on the $D$ values. For a particular $\Lambda$, the value of $D$ initially increases to reach a maximum and then starts decreasing. The graph clearly indicates that with an increase of $\Lambda$, the maximum of $D$ is red-shifted and the dispersion becomes more flatter with wavelengths, while for smaller wavelengths they are almost parallel without much change in the slope. The flatness can be understood from the following analogy: the dispersion value from the peak reduces by an amount of $25 \mathrm{ps} / \mathrm{nm} / \mathrm{km}$ for a wavelength range of $794 \mathrm{~nm}$ for $\Lambda=1.00 \mu \mathrm{m}$, while for the same values of dispersion range, the wavelength range comes out to be $760 \mathrm{~nm}$ for $\Lambda=0.85 \mu \mathrm{m}$. This can be attributed to the fact that, for higher $\Lambda$, material dispersion dominates, reducing 


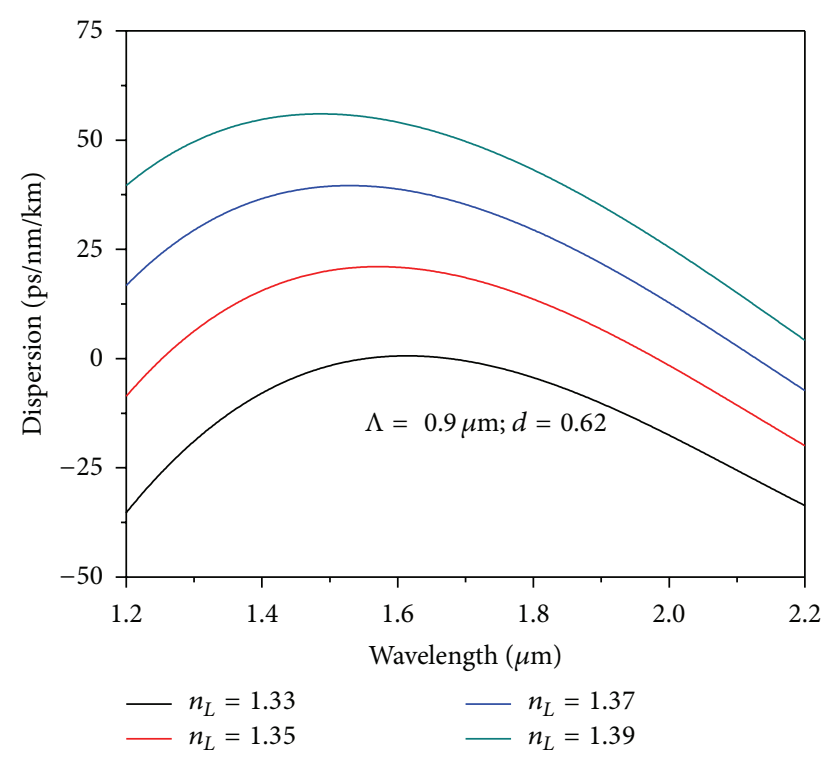

FIGURE 3: Dispersion behaviour as calculated for varying $n_{L}$ values keeping pitch $(\Lambda)$ and $d$ fixed.

the effect of waveguide dispersion. From Figure 3 it can be observed that both the magnitude and the slope of $D$ are affected for different values of $n_{L}$. An increase of $n_{L}$ causes a blue-shift of the dispersion peak and the dispersion values also increase. The effect of varying the air-hole diameter $d$ without changing the other parameters is depicted in Figure 4. It should be noted that we have changed the diameter of all the air-holes including those containing the infiltrating liquid. An interesting observation can be found out that, with an increase of $d$, the dispersion seems to be converging for higher wavelengths while the peak again gets red-shifted but with a steeper slope than that of Figure 2. The convergence can be attributed to the increased effect of waveguide dispersion to the total dispersion. Thus, the effect of varying the $\Lambda$ influences the total dispersion, whereas $d$ has the desired effect of modifying the dispersion slope, and varying $n_{L}$ modifies both the slope and dispersion.

Then, we started the second stage of the optimization procedure as the following. For this step we tried to keep $\Lambda$ nearer to $1.0 \mu \mathrm{m}$ such that we could have smaller effective area which in turn will enhance nonlinearity. Starting with $d / \Lambda=0.7$ and $n_{L}=1.35$, the values of the $\Lambda$ are changed progressively to get a near zero profile of the dispersion. To have higher values of nonlinear coefficient, we kept the $\Lambda$ values around $0.90 \mu \mathrm{m}$. Then the values of air-hole diameter and $n_{L}$ are changed progressively to either raise or lower the dispersion and change the slope. During the process we tried to keep the values of the parameters such that the values of the parameters remain practically feasible. Following the above steps, we have obtained an all-normal near zero dispersion in the wavelength around $1570 \mathrm{~nm}$ with $D$ value of $-0.41 \mathrm{ps} / \mathrm{nm} / \mathrm{km}$ with $\Lambda=0.90 \mu \mathrm{m}$ and $d=$ $0.59 \mu \mathrm{m}$ and $n_{L}=1.331$ as demonstrated in Figure 5 .

Having obtained a preliminary design using an artificial, dispersionless liquid, we continue with the third optimization

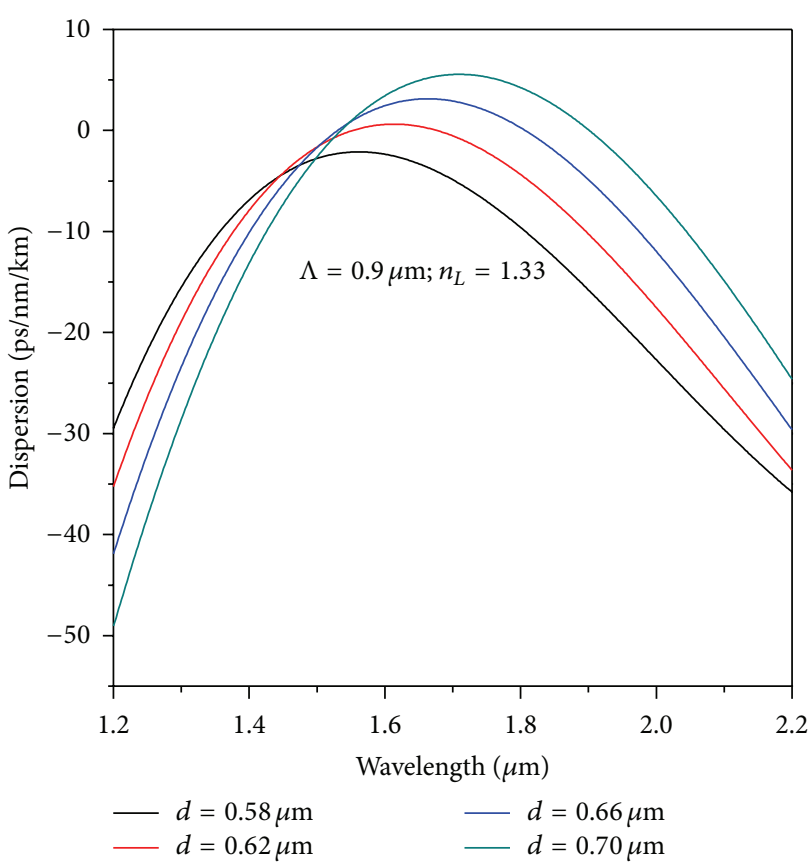

FIGURE 4: Variation of dispersion as a function of air-hole diameter $(d)$ when pitch $(\Lambda)$ and $n_{L}$ remain constant.

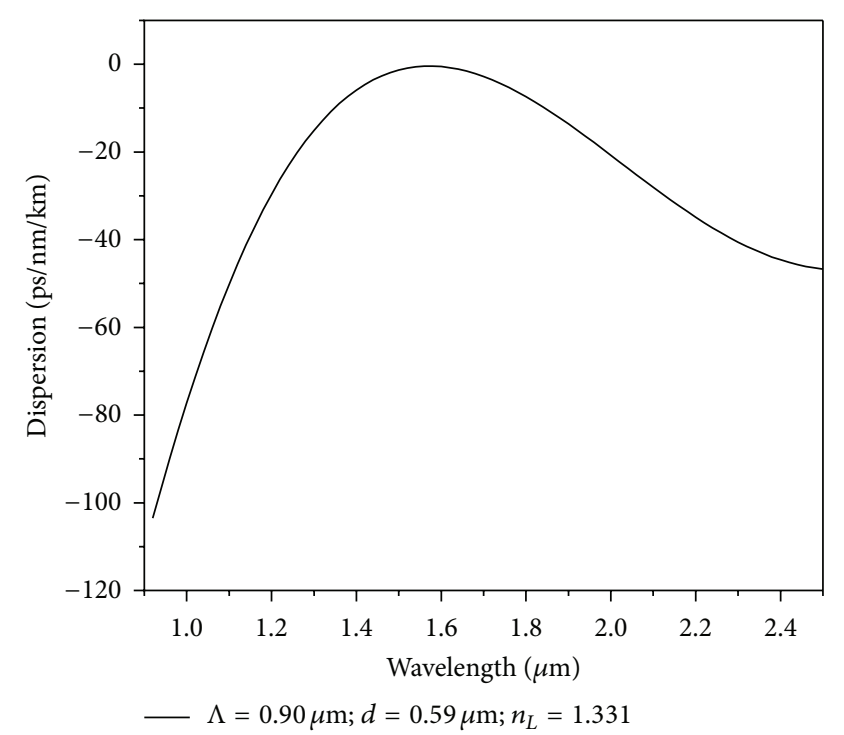

FIgURE 5: All-normal near zero dispersion with $D=$ $-0.40 \mathrm{ps} / \mathrm{nm} / \mathrm{km}$ near $1570 \mathrm{~nm}$ with $\Lambda=0.90 \mu \mathrm{m}, n_{L}=1.331$, and $d=0.59 \mu \mathrm{m}$.

stage. We select oil (calling it as oil number 1) whose RI is close to the above optimized value in the target communication wavelength band and is given by Cauchy (4) (available with M/S Cargile lab., USA [35]).

Cauchy equation for oil number 1 is known to be [35]

$$
n 1(\lambda)=1.3336794+\frac{219396}{\lambda^{2}}+\frac{3.562146 \times 10^{9}}{\lambda^{4}},
$$

where $\lambda$ are in Angstrom. 


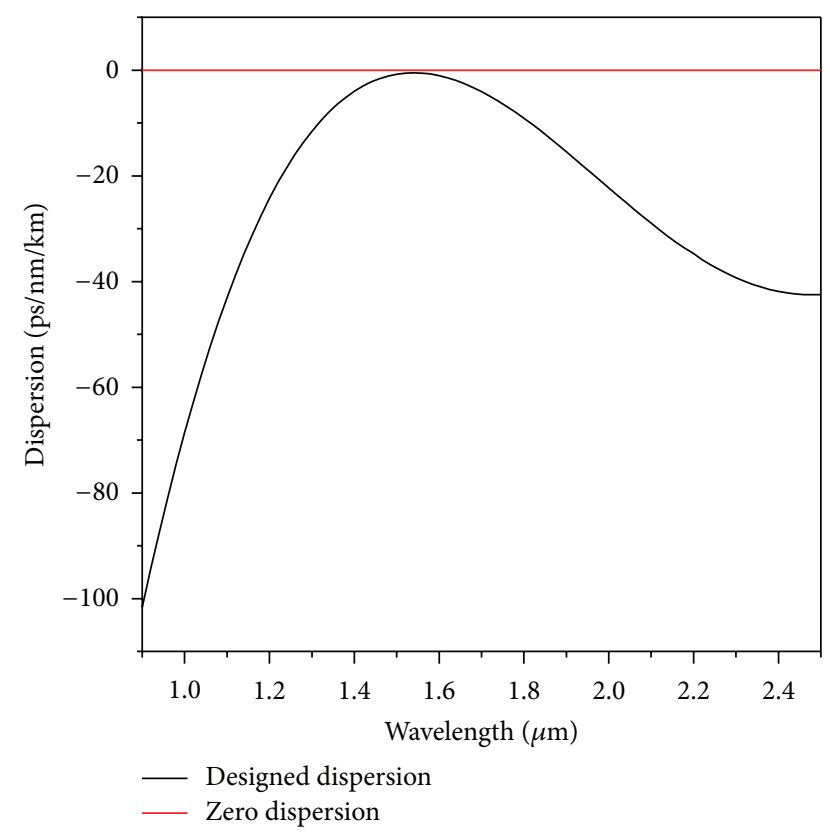

FIGURE 6: The optimized design of the all-normal near zero dispersion with $D=-0.51 \mathrm{ps} / \mathrm{nm} / \mathrm{km}$ near $1550 \mathrm{~nm}$ with $\Lambda=$ $0.90 \mu \mathrm{m}, d=0.57 \mu \mathrm{m}$ with oil \#1.

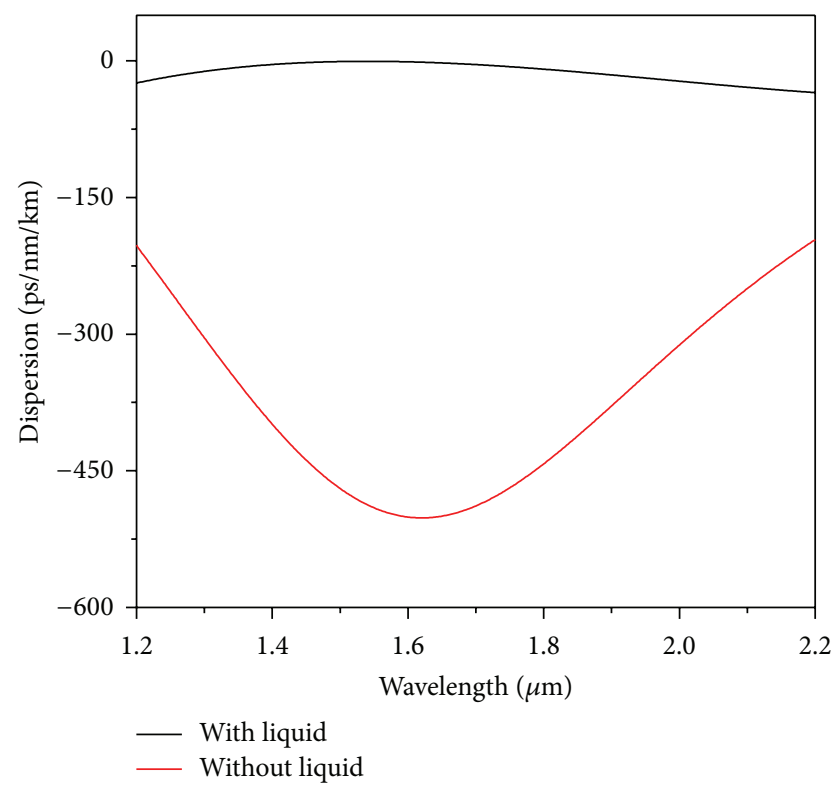

FIGURE 7: The effect of liquid infiltration in the first air-hole ring upon dispersion. Not only the dispersion values but also the slope of the graph has been drastically altered.

With this liquid an all-normal near zero dispersion PCF with $D$ value of $-0.48 \mathrm{ps} /(\mathrm{nm} . \mathrm{km})$ around $1550 \mathrm{~nm}$ of wavelength has been achieved with the designing PCF parameters of $\Lambda=0.90 \mu \mathrm{m}$ with $d=0.57 \mu \mathrm{m}$ as shown in Figure 6 . Now as we see the $d / \Lambda$ value is higher than the accepted value for endlessly "single-mode" operation. To achieve our target, we needed to make a tradeoff between a pure single-mode

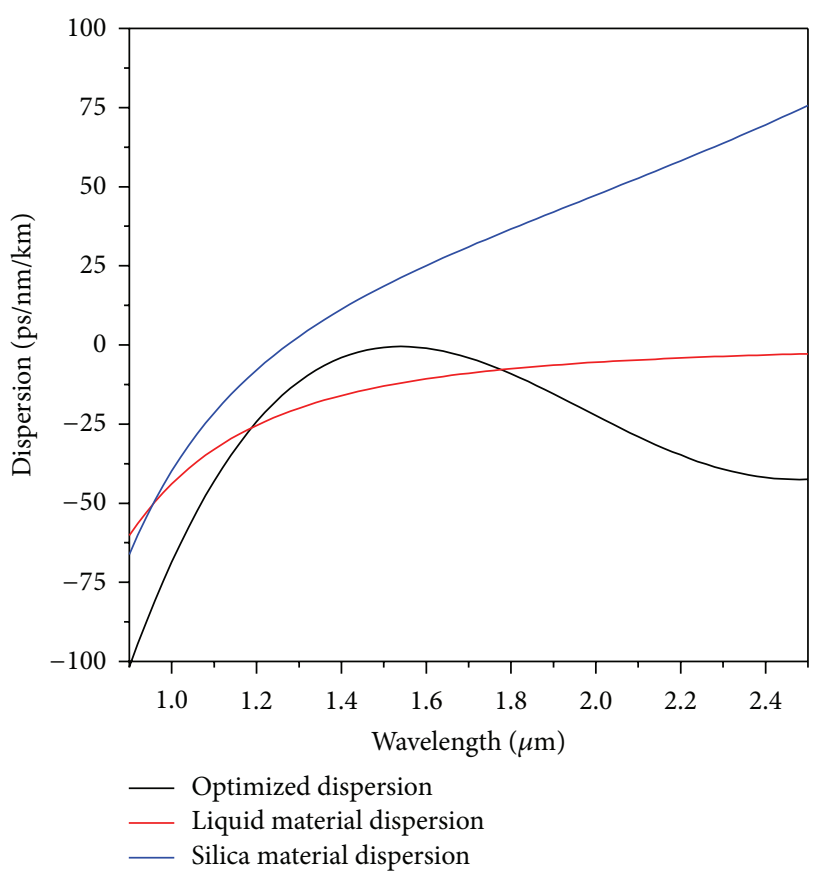

FIgURE 8: The contribution of the material dispersion of the liquid and background silica material dispersion towards the total dispersion.

guiding and few higher order mode guiding. Our numerical study reveals that with our present optimize value the next higher order mode can coexist with the fundamental one; however the confinement loss of the mode is found out to be about hundred times of magnitude higher than that of the fundamental mode throughout the spectral range considered (specially at the pumping wavelength) and hence would leak away with propagation within a very short length of the designed fiber. This would make the designed fiber an "effectively single-mode fiber." The effect of liquid infiltration upon dispersion has been presented in Figure 7. The figure clearly reveals the determining effect of the liquid infiltration in the air-holes as not only a drastic change but also a slope reversal of the dispersion could be observed. Total dispersion along with the contribution of the material dispersion of the liquid and background silica material towards the total dispersion has been shown in Figure 8 for the above optimized structure. The new control technique applied to the design of PCFs with desired dispersion properties can be widely used for specific applications such us control of SCG, sensors, and broadband transmission [1].

\section{Numerical Analysis for SCG with the Optimized Near Zero All-Normal Dispersion PCF}

NLSE, as mentioned in (2), is solved numerically by using the split step Fourier transform based beam propagation code developed by COSTP11 [36]. In our study, we have considered the pumping of laser power pulses with hyperbolic secant 


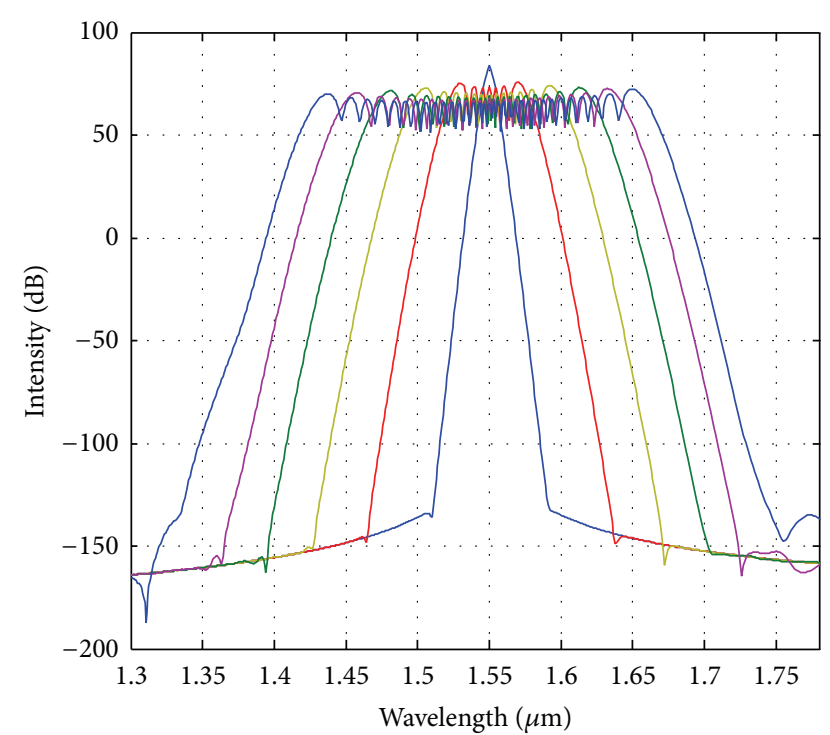

FIGURE 9: Evolution of the spectrum as the pulse propagates along the fiber. The input pulse and the output spectrum after each $25 \mathrm{~cm}$ of propagation.

field profile emerging from a laser with full width at half maximum (FWHM) of $1.5 \mathrm{ps.} \mathrm{In} \mathrm{our} \mathrm{calculations} \mathrm{we} \mathrm{set}$ the pump wavelength of $\lambda=1.55 \mu \mathrm{m}$, the closest to zero in the normal dispersion regime, in order to generate the broadest spectra. Consequently, the spectral widening is dominated by self-phase modulation (SPM) followed by optical wave breaking and the noise-sensitive soliton dynamics are suppressed by pumping in the normal dispersion region in such fibers. The soliton effect, for example, soliton fission, is completely removed, giving rise to the smooth spectra. Optimization of the all-normal dispersion fiber design allowed us to generate ultrabroadband, flat top, and smooth SC generation. An effective area of $4.03 \mu \mathrm{m}^{2}$ and nonlinear coefficient $\gamma=27.3 \mathrm{~W}^{-1} \cdot \mathrm{km}^{-1}$ are achieved in the optimized PCF with $\Lambda=0.90 \mu \mathrm{m}$ and $d=0.57 \mu \mathrm{m}$ at the pumping wavelength. The input pulse power has been kept to be $4.0 \mathrm{~kW}$ with FWHM of $1.5 \mathrm{ps}$. A possible source for such a pump can be the commercially available Er3+, Yb3+ doped silicate based fiber laser emitting around $1550 \mathrm{~nm}$ of wavelength [37]. The calculated values of higher order betas are presented in Table 1 . The spectrum evolution of the fiber after it evolves through each $25 \mathrm{~cm}$ of the fiber with the above parameters has been shown in Figure 9. The spectrum spreads for a wide wavelength range, ranging from $1300 \mathrm{~nm}$ to $1750 \mathrm{~nm}$. The intensity is calculated using the usual notation with Intensity $(\mathrm{dB})=10 \log _{10}(P)$. It can be observed that as the pulse propagates through the fiber the peak power reduces, creating new peaks on both sides of the input pulse almost symmetrically. The evolution of the spectrum as a function of propagation distance has been shown in Figure 10 which clearly shows the spreading of the pulse taking almost symmetrically around the pumping wavelength. The graph clearly indicates that after travelling a distance of 1.2 meters, the broadband spectrum spread for a wide wavelength range
TABLE 1: The values of $\beta_{n}$ used for simulation for the optimized PCF.

\begin{tabular}{lc}
\hline Higher order betas & Beta values \\
\hline$\beta_{2}\left(\mathrm{ps}^{2} / \mathrm{km}\right)$ & 0.069276 \\
$\beta_{3}\left(\mathrm{ps}^{3} / \mathrm{km}\right)$ & $-3.59804 * 10^{-3}$ \\
$\beta_{4}\left(\mathrm{ps}^{4} / \mathrm{km}\right)$ & $6.57756 * 10^{-4}$ \\
$\beta_{5}\left(\mathrm{ps}^{5} / \mathrm{km}\right)$ & $2.82896 * 10^{-6}$ \\
$\beta_{6}\left(\mathrm{ps}^{6} / \mathrm{km}\right)$ & $2.82896 * 10^{-10}$ \\
$\beta_{7}\left(\mathrm{ps}^{7} / \mathrm{km}\right)$ & $1.45745 * 10^{-12}$ \\
$\beta_{8}\left(\mathrm{ps}^{8} / \mathrm{km}\right)$ & $2.91289 * 10^{-14}$ \\
\hline
\end{tabular}

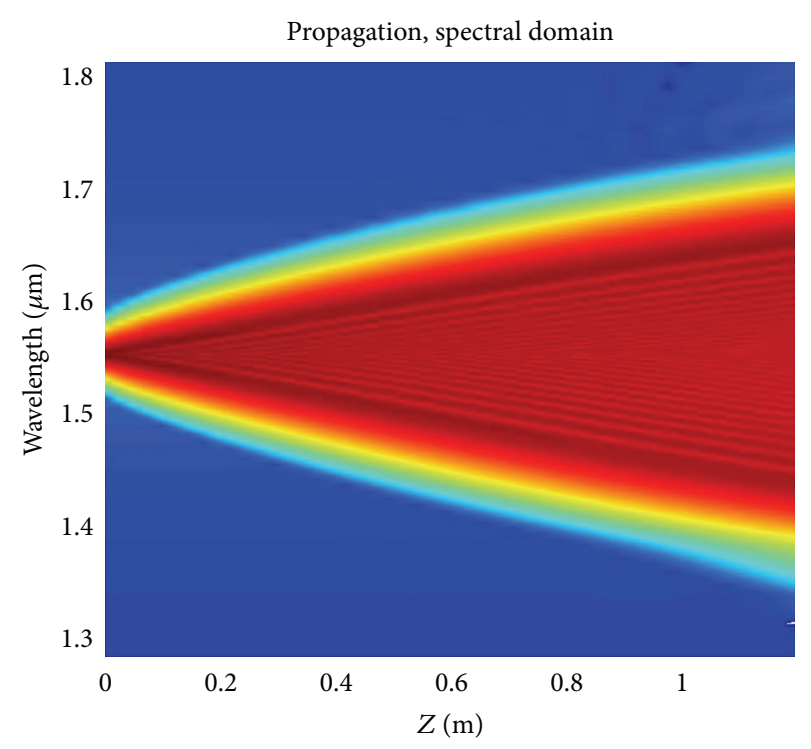

Figure 10: Propagation of the spectrum as it travels through the fiber.

from $1300 \mathrm{~nm}$ to $1750 \mathrm{~nm}$. One of the most notable features of the SC spectrum is the spectral broadening which is accompanied with an oscillating nature covering the whole spectrum. The spectrum consists of many small peaks. This particular nature is a typical pattern due to self-phase modulation (SPM), which is assumed to be dominating nonlinear effect for the spectral broadening. The multipeak structure in the system is the result of interference between the same optical frequencies in the pulse and the relatively smoother SC generation results from the combination effect of SPM and all-normal near zero flat dispersion nature of the fiber. We have calculated the spectral width of the broadband flat SC spectrum according to Begum et al. [14], where they have calculated the FWHM at the negative value of the intensity points corresponding to the peak maximum value. With the same analogy, numerical calculations show a bandwidth of $375 \mathrm{~nm}$ with our optimized fiber. However with another way, the intensity bandwidth can be calculated at $-20 \mathrm{~dB}$ from the peak point. The numerical calculation shows a $20 \mathrm{~dB}$ bandwidth of spectral width of $285 \mathrm{~nm}$ that can be achieved with a fiber length of 1.2 meter as shown in Figure 11. 


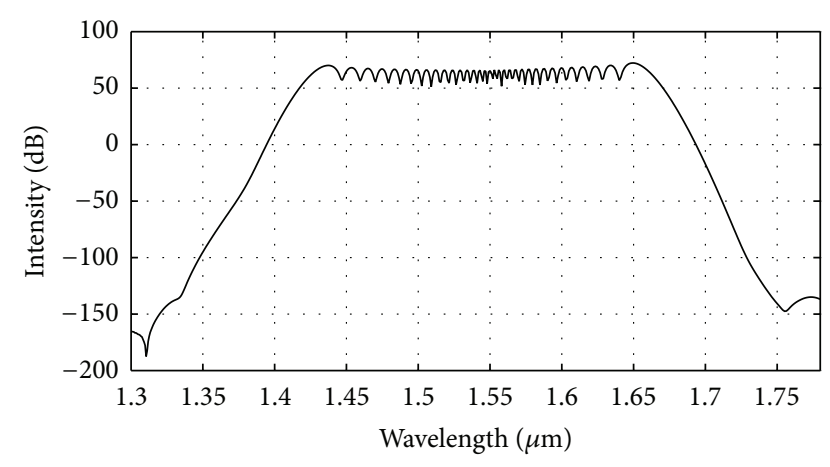

FIGURE 11: Optical spectrum with the optimized fiber after propagation of 1.2 meter. A FWHM of $375 \mathrm{~nm}$ is obtained with the optimized fiber.

\section{Tunable Properties of Inner Ring Liquid-Filled PCFs}

It is well known that the dispersion can be tailored in a PCF according to the requirement by changing the geometrical parameters. However, once a design is fixed and fabricated the dispersion of that particular fiber is also fixed. So, to have a different or optimized design we need another fiber to be fabricated. Now here comes the advantage of liquid-filled PCF. Fluid-filled PCFs have created novel opportunities for tunable dispersion control, with applications in tunable delay lines, nonlinear optics, and long period grating designs [38]. Here in this type of selective liquid infiltrated PCF, we can change the RI of the infiltrating liquid by changing the temperature of the infiltrating liquid and can tune the dispersion properties according to the requirement. As the temperature coefficient of the liquids available with $\mathrm{M} / \mathrm{s}$ Cargile lab [35] is of the order of $-4 \times 10^{-4} /{ }^{\circ} \mathrm{C}$ which is much larger than that of fused silica, the smaller changes of temperature will affect the RI of the liquid only. We have considered the optimized design shown in Figure 6 and we demonstrate the tunability property of the liquid by changing the temperature of the liquid. Figure 12 shows the dispersion characteristics for five different temperature of the liquid. It can be seen from the figure that with the increase of the temperature the peak value of the dispersion shifts away from the zero dispersion value. The peak dispersion and the corresponding variation of the peak dispersion wavelength for different temperature are demonstrated in Figure 13. The peak dispersion value with temperature shows a linear relationship with a negative slope of $-0.31084 \mathrm{ps} / \mathrm{nm} / \mathrm{km} /{ }^{\circ} \mathrm{C}$. Wavelength corresponding to peak dispersion for different temperature has been presented in Figure 13(b). The graph presents a linear relationship between the corresponding wavelength with temperature with a positive slope of $0.65 \mathrm{~nm} /{ }^{\circ} \mathrm{C}$. So we can tune the temperature of the liquid according to the requirement to obtain an optimized/required design. Our numerical calculations demonstrate that around the temperature of $23.5^{\circ} \mathrm{C}$, we could achieve an all-normal flat dispersion PCF with a peak value of $-0.089 \mathrm{ps} / \mathrm{nm} / \mathrm{km}$ around $1550 \mathrm{~nm}$ of wavelength. This design will be more demanding for the current target of all-normal flat dispersion around $1550 \mathrm{~nm}$.

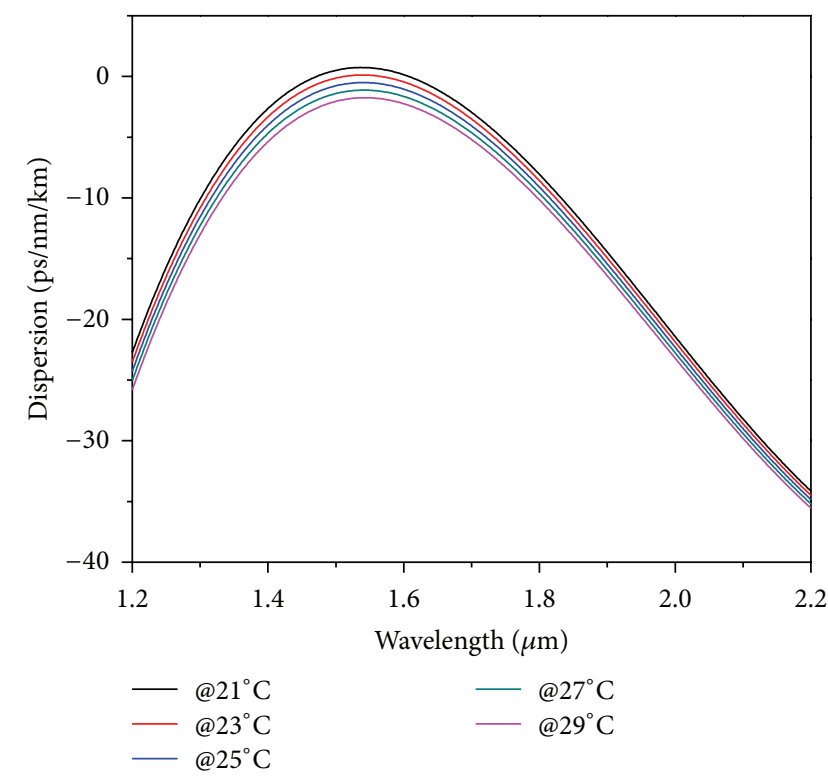

FIGURE 12: Dispersion curves for the liquid-filled PCF operated at various temperatures.

So with the tunability property of the infiltrating liquid we can easily tune the peak dispersion just by changing the temperature of the liquid for a fabricated PCF.

\section{Conclusion}

We have demonstrated a new design of all-normal near zero dispersion PCF based on selectively liquid infiltration and explored its properties for flattened broadband SC generation in near IR wavelength regions. We have studied the effect of individual PCF parameters, namely, $\Lambda$ and $d$, along with infiltrating liquid $\left(n_{L}\right)$ towards the target of all-normal near zero dispersion PCF around the communication wavelength window. Our study reveals that varying $\Lambda$ influences the total dispersion, whereas $d$ has the desired effect of modifying the dispersion slope and varying $n_{L}$ modifies both the slope and the dispersion. Based on the above findings, we have achieved an all-normal near zero dispersion PCF with $D$ value of $-0.48 \mathrm{ps} /(\mathrm{nm} \cdot \mathrm{km})$ around $1550 \mathrm{~nm}$ of wavelength with the designing PCF parameters of $\Lambda=0.90 \mu \mathrm{m}$ with $d=$ $0.57 \mu \mathrm{m}$ and oil number 1 . Our study reveals that by adjusting the different parameters, near ZDW can be tuned according to the requirement. With the above designed PCF, we have obtained a smooth and broadband spectra ranging from $1350 \mathrm{~nm}$ to $1725 \mathrm{~nm}$, that is, for a bandwidth of $375 \mathrm{~nm}$ with 1.2 meter length of the fiber. We have also demonstrated the tunability properties of the optimized PCF. The peak wavelength corresponding to all-normal flat dispersion can be well tuned by the refractive index of the liquid which can be controlled by the operation temperature. By changing the operational temperature to $23.5^{\circ} \mathrm{C}$ we could achieve a peak dispersion of $-0.089 \mathrm{ps} / \mathrm{nm} / \mathrm{km}$ around $1550 \mathrm{~nm}$ of wavelength with our optimized design. The tunability of the liquid-filled PCF can be utilized for wide wavelength range applications and also 


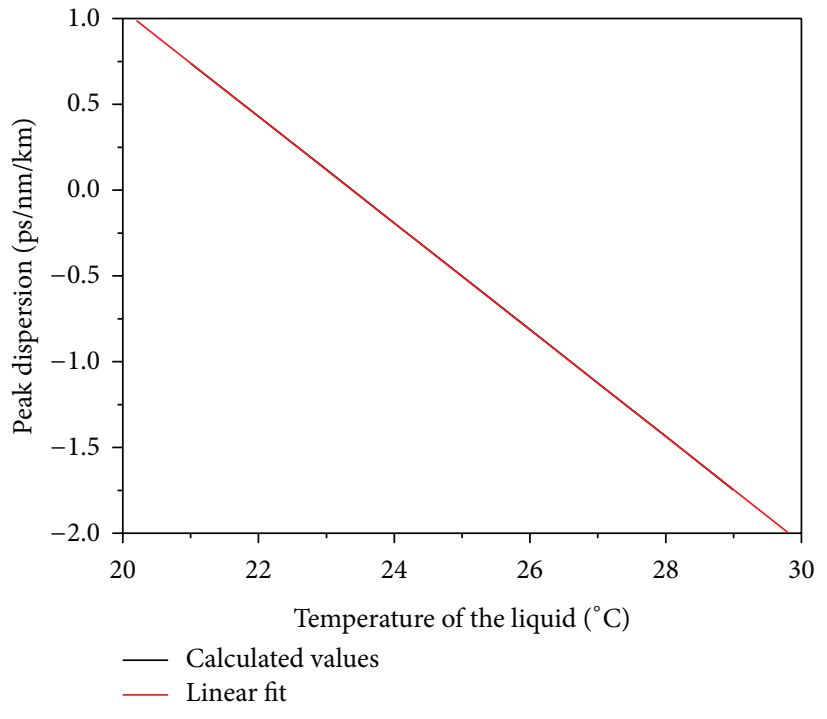

(a)

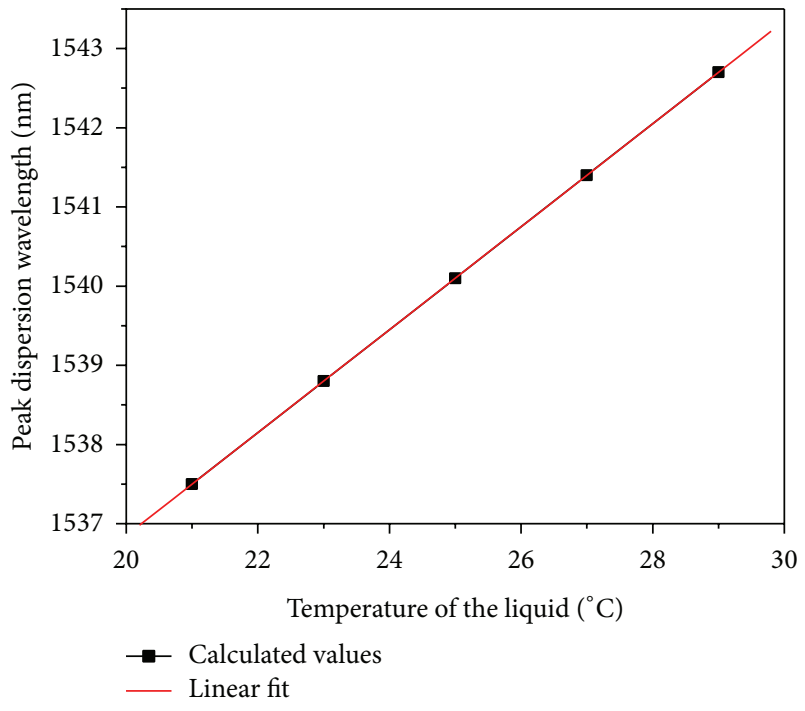

(b)

FIGURE 13: (a) Peak dispersion values of the liquid-filled PCF operated at variant temperatures. (b) Peak dispersion wavelength of the liquidfilled PCF operated at variant temperatures.

to correct possible fabrication mismatches in the geometry of PCF structures. The significance of this work is that it provides a new type of dispersion engineered silica based PCF for near Infrared (IR) SC source with flat broadband spectrum with about a meter long of the fiber which can be useful for applications in metrology, spectroscopy, and optical coherence tomography in the near infrared region.

\section{Conflict of Interests}

The authors declare that there is no conflict of interests regarding the publication of this paper.

\section{Acknowledgments}

The authors would like to thank Dr. Boris Kuhlmey, University of Sydney, Australia, for providing valuable suggestions in understanding the software for designing and studying the properties of different structures. Thanks are also due to Dr. Alessandro Tonello of Xlim Institute, UMR CNRS Limoges Cedex, France, for providing valuable suggestion in developing and understanding the NLSE codes. The authors acknowledge sincerely the Defence Research and Development Organization, Govt. of India and CRF of IIT Kharagpur for the financial support to carry out this research.

\section{References}

[1] J. M. Dudley, G. Genty, and S. Coen, "Supercontinuum generation in photonic crystal fiber," Reviews of Modern Physics, vol. 78, no. 4, pp. 135-184, 2006.

[2] J. Swiderski, F. Théberge, M. Michalska, P. Mathieu, and D. Vincent, "High average power supercontinuum generation in a fluoroindate fiber," Laser Physics Letters, vol. 11, pp. 015106015109, 2014.

[3] G. Sobon, M. Klimczak, J. Sotor et al., "Infrared supercontinuum generation in soft-glass photonic crystal fibers pumped at 1560 nm," Optical Materials Express, vol. 4, pp. 7-15, 2014.

[4] A. di Tommaso, M. de Sario, L. Mescia, and F. Prudenzano, "Numerical analysis of aperiodic photonic crystal fiber structures for supercontinuum generation," Optical Engineering, vol. 51, pp. 0350031-0350037, 2012.

[5] J. Broeng, D. Mogilevstev, S. E. Barkou, and A. Bjarklev, "Photonic crystal fibers: a new class of optical waveguides," Optical Fiber Technology, vol. 5, no. 3, pp. 305-330, 1999.

[6] P. S. J. Russell, "Photonic-crystal fibers," Journal of Lightwave Technology, vol. 24, no. 12, pp. 4729-4749, 2006.

[7] K. Saitoh, N. J. Florous, and M. Koshiba, "Theoretical realization of holey fiber with flat chromatic dispersion and large mode area: an intriguing defected approach," Optics Letters, vol. 31, no. 1, pp. 26-28, 2006.

[8] N. Florous, K. Saitoh, and M. Koshiba, "The role of artificial defects for engineering large effective mode area, flat chromatic dispersion, and low leakage losses in photonic crystal fibers: towards high speed reconfigurable transmission platforms," Optics Express, vol. 14, no. 2, pp. 901-913, 2006.

[9] K. Saitoh and M. Koshiba, "Highly nonlinear dispersionflattened photonic crystal fibers for supercontinuum generation in a telecommunication window," Optics Express, vol. 12, no. 10, pp. 2027-2032, 2004.

[10] K. Saitoh, M. Koshiba, T. Hasegawa, and E. Sasaoka, "Chromatic dispersion control in photonic crystal fibers: application to ultra-flattened dispersion," Optics Express, vol. 11, no. 8, pp. 843852, 2003.

[11] F. Poletti, V. Finazzi, T. M. Monro, N. G. R. Broderick, V. Tse, and D. J. Richardson, "Inverse design and fabrication tolerances of ultra-flattened dispersion holey fibers," Optics Express, vol. 13, no. 10, pp. 3728-3736, 2005. 
[12] T.-L. Wu and C.-H. Chao, "A novel ultraflattened dispersion photonic crystal fiber," IEEE Photonics Technology Letters, vol. 17, no. 1, pp. 67-69, 2005.

[13] X. Zhao, G. Zhou, L. Shuguang et al., "Photonic crystal fiber for dispersion compensation," Applied Optics, vol. 47, no. 28, pp. 5190-5196, 2008.

[14] F. Begum, Y. Namihira, T. Kinjo, and S. Kaijage, "Supercontinuum generation in square photonic crystal fiber with nearly zero ultra-flattened chromatic dispersion and fabrication tolerance analysis," Optics Communications, vol. 284, no. 4, pp. 965-970, 2011.

[15] C. Zhang, G. Kai, Z. Wang et al., "Transformation of a transmission mechanism by filling the holes of normal silica-guiding microstructure fibers with nematic liquid crystal," Optics Letters, vol. 30, no. 18, pp. 2372-2374, 2005.

[16] T. T. Alkeskjold, J. Lægsgaard, A. Bjarklev et al., "Highly tunable large-core single-mode liquid-crystal photonic bandgap fiber," Applied Optics, vol. 45, no. 10, pp. 2261-2264, 2006.

[17] B. J. Eggleton, C. Kerbage, P. S. Westbrook, R. S. Windeler, and A. Hale, "Microstructured optical fiber devices," Optics Express, vol. 9, no. 13, pp. 698-713, 2001.

[18] C. Martelli, J. Canning, K. Lyytikainen, and N. Groothoff, "Water-core fresnel fiber," Optics Express, vol. 13, no. 10, pp. 3890-3895, 2005.

[19] S. Yiou, P. Delaye, A. Rouvie et al., "Stimulated Raman scattering in an ethanol core microstructured optical fiber," Optics Express, vol. 13, no. 12, pp. 4786-4791, 2005.

[20] C.-P. Yu and J.-H. Liou, "Selectively liquid-filled photonic crystal fibers for optical devices," Optics Express, vol. 17, no. 11, pp. 8729-8734, 2009.

[21] K. M. Gundu, M. Kolesik, J. V. Moloney, and K. S. Lee, "Ultraflattened-dispersion selectively liquid-filled photonic crystal fibers," Optics Express, vol. 14, no. 15, pp. 6870-6878, 2006.

[22] C. P. Yu, J. H. Liou, S. S. Huang, and H. C. Chang, "Tunable dual-core liquid-filled photonic crystal fibers for dispersion compensation," Optics Express, vol. 16, no. 7, pp. 4443-4451, 2008.

[23] L. Xiao, W. Jin, M. S. Demokan, H. L. Ho, Y. L. Hoo, and C. Zhao, "Fabrication of selective injection microstructured optical fibers with a conventional fusion splicer," Optics Express, vol. 13, no. 22, pp. 9014-9022, 2005.

[24] A. Witkowska, K. Lai, S. G. Leon-Saval, W. J. Wadsworth, and T. A. Birks, "All-fiber anamorphic core-shape transitions," Optics Letters, vol. 31, no. 18, pp. 2672-2674, 2006.

[25] W. H. Reeves, J. C. Knight, P. S. J. Russell, and P. J. Roberts, "Demonstration of ultra-flattened dispersion in photonic crystal fibers," Optics Express, vol. 10, no. 14, pp. 609-613, 2002.

[26] E. W. Washburn, "The dynamics of capillary flow," Physical Review, vol. 17, pp. 273-283, 1921.

[27] J. H. Liou, S. S. Huang, and C. P. Yu, "Loss-reduced highly birefringent selectively liquid-filled photonic crystal fibers," Optics Communications, vol. 283, no. 6, pp. 971-974, 2010.

[28] N. Vukovic, N. Healy, and A. C. Peacock, "Guiding properties of large mode area silicon microstructured fibers: a route to effective single mode operation," Journal of the Optical Society of America B: Optical Physics, vol. 28, no. 6, pp. 1529-1533, 2011.

[29] N. Healy, J. R. Sparks, R. R. He, P. J. A. Sazio, J. V. Badding, and A. C. Peacock, "High index contrast semiconductor ARROW and hybrid ARROW fibers," Optics Express, vol. 19, no. 11, pp. 10979-10985, 2011.
[30] Y. Miao, B. Liu, K. Zhang, Y. Liu, and H. Zhang, "Temperature tunability of photonic crystal fiber filled with $\mathrm{Fe}_{3} \mathrm{O}_{4}$ nanoparticle fluid," Applied Physics Letters, vol. 98, no. 2, pp. 021103021105, 2011.

[31] M.-Y. Zhang, S.-G. Li, Y.-Y. Yao, B. Fu, and L. Zhang, "A dark hollow beam from a selectively liquid-filled photonic crystal fibre," Chinese Physics B, vol. 19, no. 4, pp. 047103-047106, 2010.

[32] W. Qian, C.-L. Zhao, J. Kang, X. Dong, Z. Zhang, and S. Jin, "A proposal of a novel polarizer based on a partial liquid-filled hollow-core photonic bandgap fiber," Optics Communications, vol. 284, no. 19, pp. 4800-4804, 2011.

[33] G. P. Agrawal, Nonlinear Fiber Optics, Optics and Photonics Series, Academic Press, San Diego, Calif, USA, 4th edition, 2007.

[34] Q. Lin and G. P. Agrawal, "Raman response function for silica fibers," Optics Letters, vol. 31, no. 21, pp. 3086-3088, 2006.

[35] http://www.cargille.com/.

[36] http://www.ufe.cz/costp11/.

[37] D. Jackson, "Towards high-power mid-infrared emission from a fibre laser," Nature Photonics, vol. 6, pp. 423-431, 2012.

[38] B. T. Kuhlmey, B. J. Eggleton, and D. K. C. Wu, "Fluid-filled solid-core photonic bandgap fibers," The Journal of Lightwave Technology, vol. 27, pp. 1617-1630, 2009. 

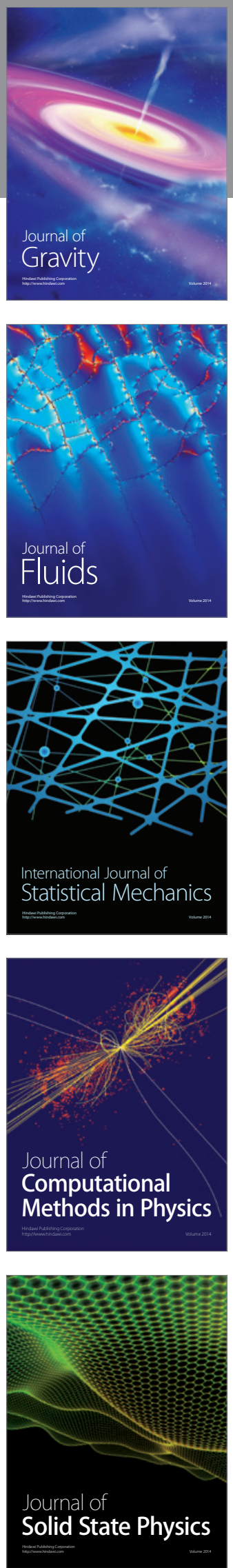

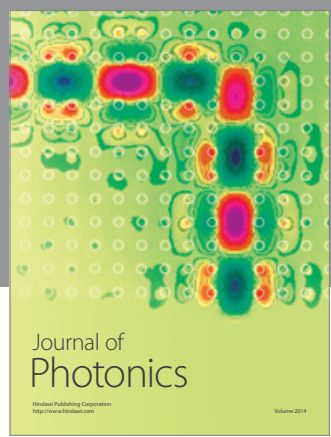

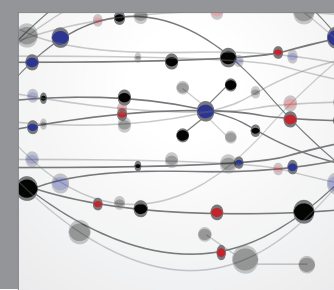

The Scientific World Journal

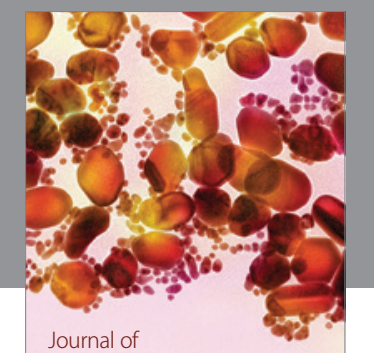

Soft Matter
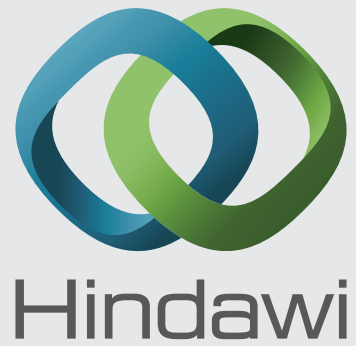

Submit your manuscripts at

http://www.hindawi.com
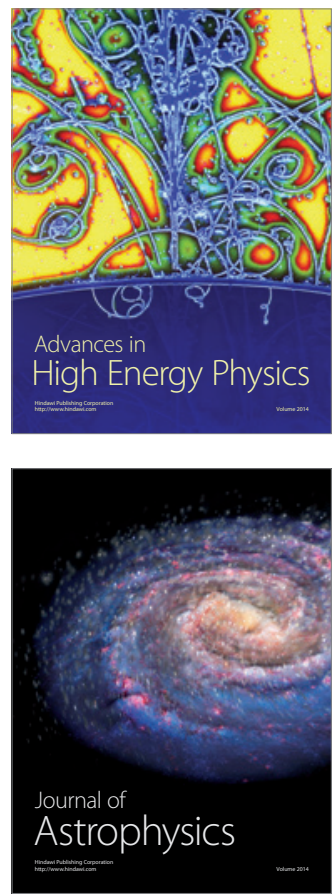
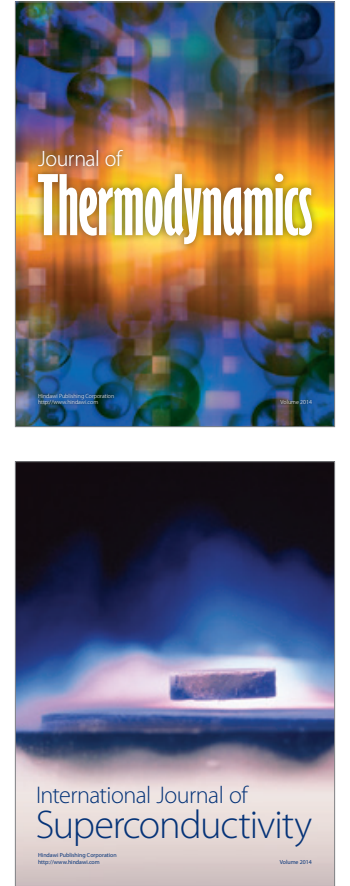
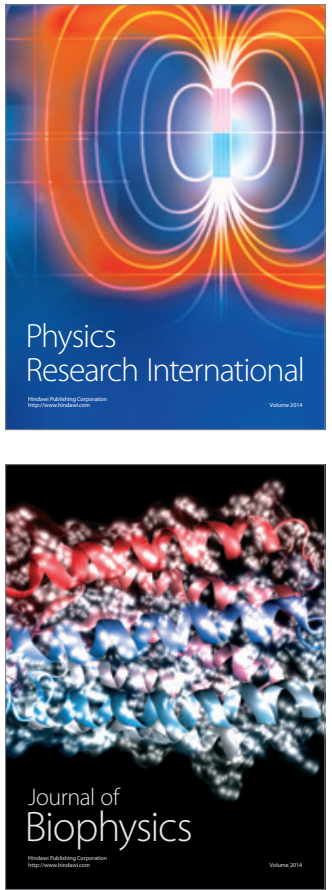
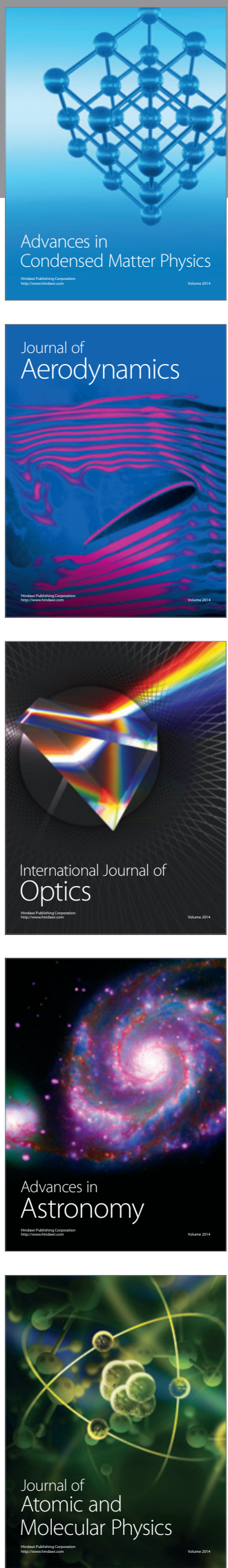